\title{
1808-1814 : démographie et guerre en Espagne
}

\section{Esteban Canales}

\section{(2) OpenEdition}

\section{Journals}

\section{Édition électronique}

URL : https://journals.openedition.org/ahrf/1663

DOI : 10.4000/ahrf.1663

ISSN : 1952-403X

Éditeur :

Armand Colin, Société des études robespierristes

Édition imprimée

Date de publication : 1 juin 2004

Pagination : 37-52

ISSN : 0003-4436

\section{Référence électronique}

Esteban Canales, «1808-1814: démographie et querre en Espagne». Annales historiques de la Révolution française [En ligne], 336 | avril-juin 2004, mis en ligne le 15 juillet 2007, consulté le 23 avril 2022. URL : http://journals.openedition.org/ahrf/1663 ; DOI : https://doi.org/10.4000/ahrf.1663

Ce document a été généré automatiquement le 23 avril 2022.

Tous droits réservés 


\title{
1808-1814: démographie et guerre en Espagne
}

\author{
Esteban Canales
}

1 La guerre qui ravagea le territoire espagnol entre 1808 et 1814 a fait l'objet de nombreuses études, mais très peu se sont appliquées à analyser son prix en vies humaines et son impact sur la démographie du pays. Cette lacune, qu'explique la difficulté à exploiter des sources nombreuses, mais éparses, entretient des affirmations assez floues sur l'impact démographique du conflit, et particulièrement sur l'importance du taux de mortalité. Cet article a pour but de combler en partie cette lacune, en étudiant les caractères et l'importance de la crise provoquée par la guerre, en effectuant une estimation des pertes humaines subies à l'époque du conflit, en examinant leur distribution dans le temps et dans l'espace, et en évaluant l'impact de ces années sur l'évolution de la population espagnole ${ }^{1}$.

2 En Espagne, l'absence de recensement fiable dans les années qui précédèrent et suivirent la guerre empêche de recourir à ce moyen pour mesurer l'étendue des pertes en vies humaines pendant le conflit de 1808-1814. Le premier recensement crédible du XIXe siècle n'a été effectué que tardivement, en 1857, et se révèle ainsi peu utile pour cette étude, même si la distribution de la population par âge y laisse entrevoir les cicatrices occasionnées par le conflit, les générations qui étaient nées ou avaient vécu durant ces années étant beaucoup moins représentées que ce que l'on pouvait espérer (cf. infra, graphique 3). Mais cette diminution des effectifs est aussi due à la crise du début du siècle. La guerre, en effet, fut précédée d'une crise démographique, qui ne permet pas aux recensements antérieurs à 1808 (1787, 1797) d'indiquer de manière fiable le nombre des habitants vivant en Espagne au début des affrontements. Dans de telles conditions, les registres paroissiaux deviennent la seule alternative valide pour connaître l'impact démographique de la guerre de 1808-1814, et ce malgré leurs lacunes, les manques créés par une mauvaise préservation et la représentation géographique inégale des séries connues. Pour estimer la mortalité pendant ces années de guerre, la comparer avec celle de la crise précédente et préciser sa distribution dans le temps et dans l'espace, j'ai tenté d'utiliser le plus grand nombre possible de ces séries 
de registres. Les résultats apparaissent sur les cartes 1 et 2 , ainsi que sur le graphique 1 . Ils démontrent que, pendant la guerre, les pointes de mortalité tendent à se concentrer en 1809 et 1812 ; l'intensité de la crise des années de guerre, d'un impact très inégal sur les différents territoires de la péninsule, va de pair avec celle des premières années du siècle à laquelle elle fait suite pratiquement sans aucune interruption.

Dans la majorité des zones géographiques pour lesquelles je dispose d'informations, l'année de mortalité la plus élevée pendant la guerre fut soit 1809, soit 1812, avec comme unique exception significative la zone Pays Basque-Navarre. Une certaine cohérence s'observe dans la distribution géographique de cette mortalité : les maxima de 1809 se produisent surtout dans le tiers nord de la péninsule (Galice, Cantabria, Asturies, Rioja, Catalogne), alors que ceux de 1812 tendent à se concentrer dans certaines zones du tiers sud (Andalousie et provinces méridionales de Valence), le centre de la péninsule (Plateau de Castille et Estrémadure) constituant un territoire moins défini ; dans l'exception que constituent le Pays Basque et la Navarre, les taux de mortalité les plus élevés correspondent tous à 1813. Les différents travaux sur lesquels se base cet article fournissent quelques pistes pour comprendre cette distribution géographique de la mortalité.

Carte 1. Pointes de mortalité, 1808-1814²

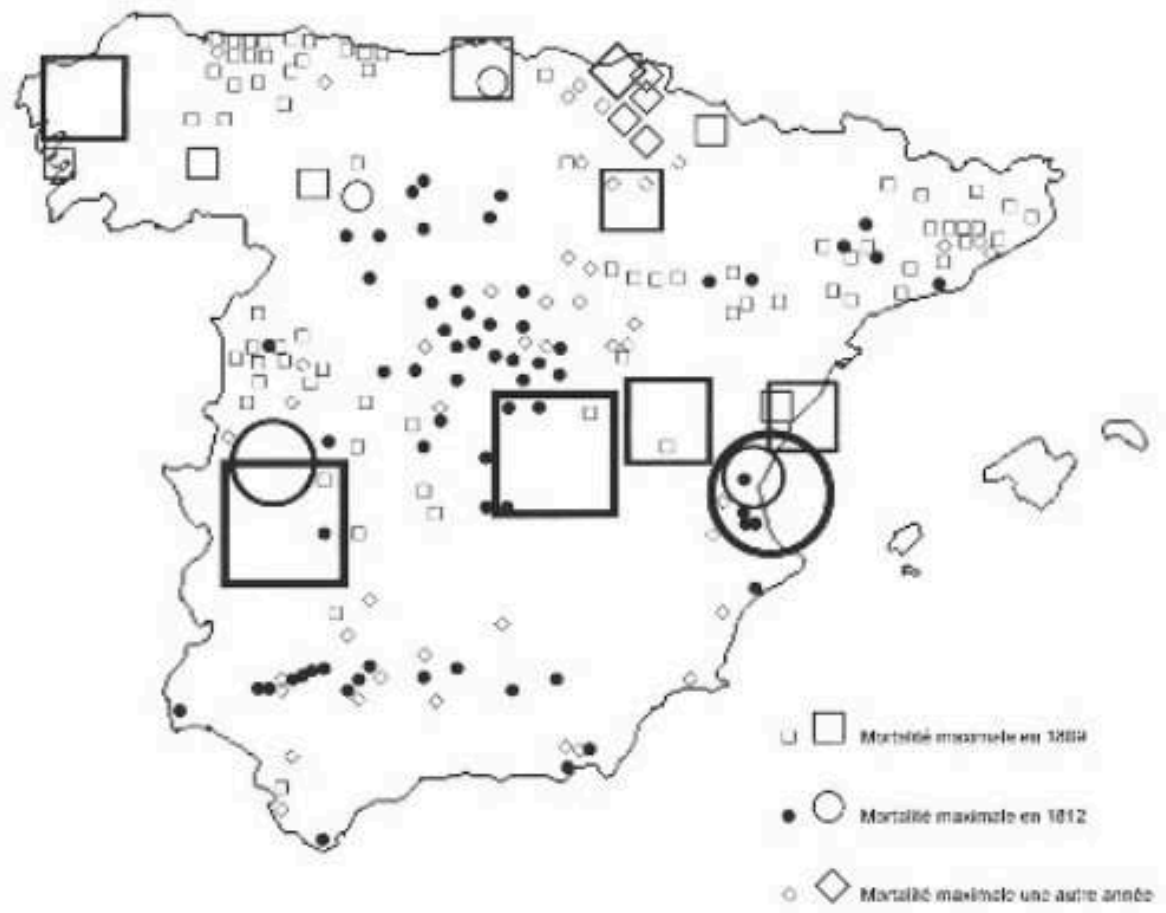

Différents facteurs permettent d'expliquer la crise de surmortalité de 1809, parmi lesquels la guerre, qui joue un rôle important dans son déclenchement et son aggravation : les pertes de récoltes dues à leur destruction, à leur réquisition ou à la fuite de la population, les suites de la transformation du territoire en terrain d'affrontement, et les épidémies dont la diffusion est facilitée par les mouvements de troupes et de civils, sont autant d'éléments qui expliquent les maxima de mortalité observés en Catalogne, en Galice et aux Asturies. Dans toutes ces régions, 1809 est l'année où le conflit armé atteint une violence particulière, après l'entrée de nouvelles 
troupes napoléoniennes dans la péninsule lors des derniers mois de 1808: c'est la mobilisation autour de la défense de Gérone, et sa chute (Catalogne) ; c'est l'occupation des Asturies; c'est l'invasion française en Galice et le soulèvement populaire qu'elle provoque. En Galice, à l'abri des grandes crises de subsistances grâce à sa diversité agricole, la crise de 1809 fut peut-être la plus importante de toutes celles que connut la province lors des deux siècles précédents ${ }^{3}$. En Catalogne, où l'année 1809 fut également la plus catastrophique, la situation de guerre, avec les disettes et les épidémies qu'elle produit, fut la cause des fortes pertes de population pour des communes comme Gérone $^{4}$, victime d'un long siège, Terrassa ${ }^{5}$, où la disette et le typhus succédèrent aux destructions commises par les Français, et la zone de la Plana de Vic ${ }^{6}$ où, après l'arrivée des troupes françaises, se propagea une épidémie de typhus exanthématique. L'année 1809 fut aussi la pire de la guerre dans certaines parties de Castille-Léon, où les mouvements de troupes et les combats furent importants : dans les régions du Léon de Bierzo et de la Maragatería, théâtre de la débâcle désordonnée des Anglais vers La Corogne et de l'arrivée des armées napoléoniennes, et sur les terres limitrophes du Portugal. Dans toutes ces régions, comme dans d'autres zones des deux tiers nord et centre de la péninsule qui n'ont pas, elles non plus, échappé à la rigueur de la guerre, les pertes directement produites par le conflit (patriotes morts au combat ou victimes des représailles françaises) ne semblent pas être les plus importantes. Cependant, parmi les régions étudiées, la crise de subsistances n'a eu un rôle dominant qu'en Cantabria, où le prix des aliments atteignit son maximum séculaire en $1809^{7}$; mais les mauvaises récoltes qui furent à l'origine de cette crise s'étaient produites dans un contexte de désordre créé par la guerre.

5 Après deux ans d'un certain redressement -en 1810 et 1811, les épidémies diminuent et il n'y a pas de grandes pénuries, malgré la guerre qui continue- l'année 1812 connaît à nouveau une crise qui, généralement, fut moins sévère que celle de 1809 . Contrairement à ce qui s'était produit trois ans plus tôt, la crise de subsistances joue désormais un rôle essentiel, bien qu'il ne faille pas oublier l'importance perturbatrice du contexte de guerre. La plupart des séries de prix du blé recueillies par Anes atteignent leurs valeurs maximales cette année là, dépassant celles de $1804^{8}$. La forte augmentation des prix, y compris dans des régions côtières telles qu'Alicante ou le littoral barcelonais, ou encore en un point stratégique comme celui de la capitale de la monarchie, laisse à penser qu'à cette occasion, contrairement à 1804, la désarticulation des marchés, les tensions dans la demande d'aliments et les destructions occasionnées par la guerre eurent une grande importance. La faim accompagna inévitablement des prix si élevés et se fit ressentir en de nombreux endroits, favorisant les taux élevés de mortalité enregistrés dans l'ensemble de la région valencienne ${ }^{9}$, dans de larges zones des deux plateaux et dans la capitale, Madrid, où elle atteignit des dimensions catastrophiques ${ }^{10}$. Dans certains endroits de Catalogne, où l'intensité exceptionnelle de la crise de 1809 fait ombrage aux taux de mortalité de 1812, cette année là fut connue sous le nom d'«any de la fam», l'année de la faim. Mais cette crise ne fut pas accompagnée, comme en 1804, d'éruptions épidémiques de grande ampleur, excepté peut-être dans la région de Murcie, où les villes de Carthagène et de Murcie furent assaillies par la fièvre jaune ${ }^{11}$. Ceci expliquerait pourquoi la mortalité n'atteint pas des chiffres plus élevés, mais ne nous éclaire pas pour autant sur les raisons de l'inégal impact géographique de la crise de 1812. Il y a de nombreux éléments à prendre en compte pour comprendre la diversité de ses répercussions : l'intensité plus élevée de la crise de 1809, qui relèguerait la crise suivante au second plan dans les endroits où elle 
avait été la plus dure (Catalogne, Asturies, Galice) ; une agriculture plus diversifiée qui permettrait d'échapper, jusqu'à un certain degré, aux ravages d'une mauvaise récolte (Galice et la façade du Cantabrique) et une situation éloignée des fronts de la guerre (là encore, la Galice).

Graphique 1. Index d'évolution de la mortalité, 1800-181512

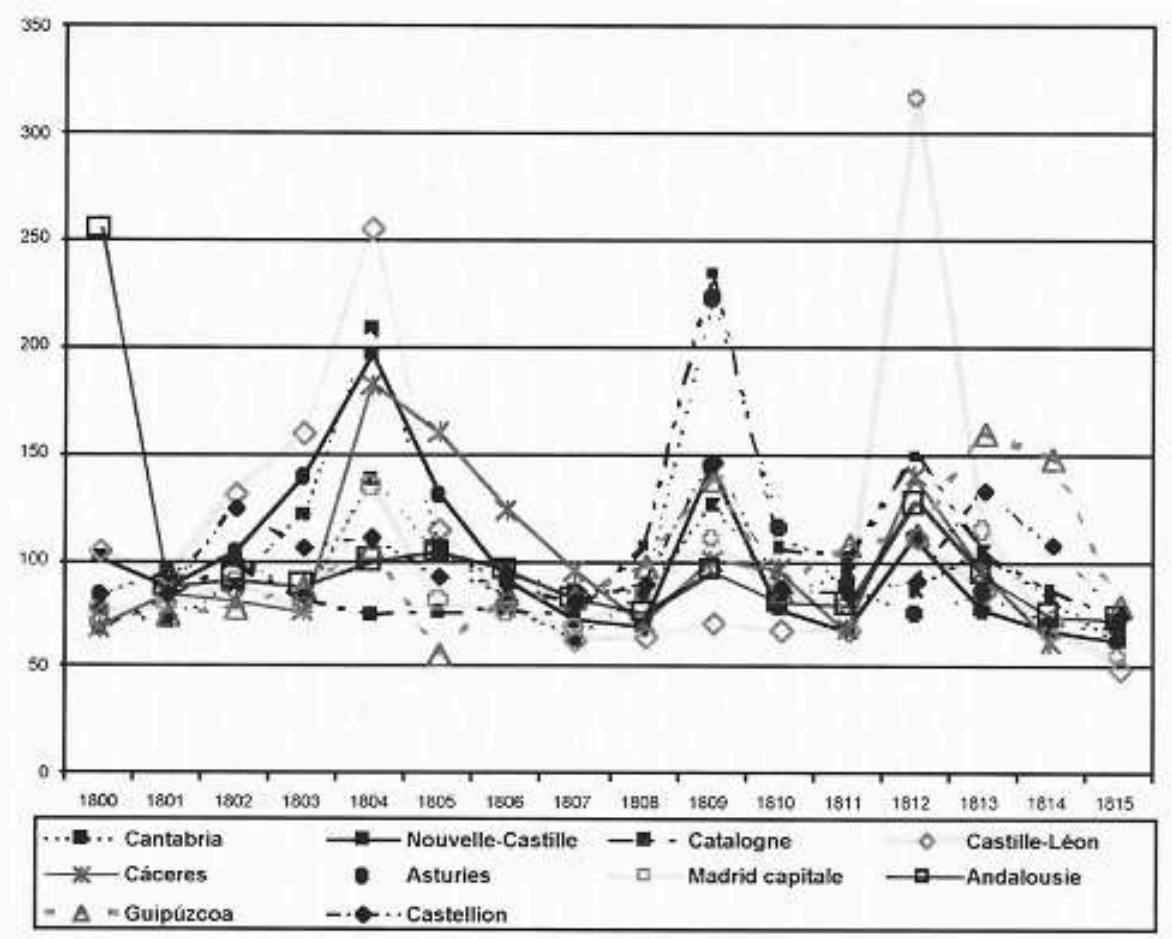

7 Pour la plupart des zones pour lesquelles on dispose de données, l'année 1813 voit la mortalité décliner, sauf dans la province de Castellion, où il se peut que l'augmentation observée soit liée à la retraite des troupes françaises vers la Catalogne, sous le commandement de Suchet, et surtout dans le Pays Basque et la Navarre. Dans beaucoup de régions de la zone Pays Basque-Navarre, tout comme dans l'ensemble de la province de Guipúzcoa, 1813 est l'année où la mortalité atteint son maximum, depuis la crise précédente du milieu de la décennie 1790, née elle aussi dans un contexte de guerre. Comme à cette époque, c'est « l'épuisement économique produit par la guerre dû aux réquisitions, matériel d'équipement et vivres et la situation parallèle de pénurie de grain » qui apparaît comme le facteur dominant dans la vallée du Bastan et aussi, probablement, dans les autres zones où l'on observe des taux élevés de mortalité : La Merindad de Estella, la région de la Barranca, les Cinco Villas, la plupart des paroisses de Guipúzcoa et d'autres localités du Pays Basque. Mais il faut aussi préciser qu'il s'agit d'une crise mineure, d'intensité inférieure aux crises ayant dévasté les territoires péninsulaires en 1809 ou en 1812.

Comme nous l'avons déjà indiqué, la crise de mortalité des années de la guerre se produit pratiquement immédiatement après la crise du début du siècle (1800-1805). Les données introduites dans la deuxième carte (voir page suivante), qui situe géographiquement l'année de mortalité la plus forte de la période 1800-1814, et dans la graphique 1, qui transcrit l'évolution des taux de mortalité de douze régions pendant 
les années mentionnées ci-dessus, permettent d'ébaucher une comparaison entre les deux crises avec les résultats suivants: 1)aucune crise particulière n'a dominé le territoire péninsulaire espagnol durant cette période: les années les plus critiques se situent dans l'une ou l'autre des deux étapes de cette période, de sorte qu'on ne peut assigner de suprématie particulière ni aux années du début du siècle, ni à celles de la Guerre d'Indépendance. 2) En Andalousie et à l'intérieur des terres espagnoles, la crise s'est concentrée dans les années antérieures à 1808, avec comme seule exception significative connue celle de la ville de Madrid. 3) En Galice, sur la façade atlantique septentrionale et en Méditerranée, la plus forte intensité se fit ressentir dans les années qui suivirent, qui furent celles des moments de guerre.

9 En examinant les diverses études, soutenues par la carte et le graphique, on peut affirmer que la période qui frappa la population sous forme d'épidémies ou/et de crise de subsistance depuis 1800 , et plus précisément entre 1803 et 1805 , fut d'un intensité égale ou supérieure à celle de la Guerre d'Indépendance; bien que pendant les deux crises il y eut des maxima de mortalité assez proches, ils eurent une étendue supérieure dans la première période, où elle comprit de larges parties des deux Castilles ainsi que d'autres régions d'Espagne centrale. Quant à la crise des années de guerre, son amplitude géographique fut légèrement supérieure à celle de la précédente. La crise initiale s'étendit, elle aussi, au-delà de l'Espagne centrale et de l'Andalousie, mais dans une moindre mesure et de façon un peu plus atténuée que la seconde, qui se fit sentir sous forme de maxima secondaires dans une grande partie des zones affectées par la crise de début de siècle. De manière générale, les effets des deux crises sur la population durent être similaires. Toutes deux se sont prolongées sur plusieurs années, ce qui rallongea les effets des crises ponctuelles $(1803,1804,1809$ et 1812) et empêcha tout recouvrement à court terme; comme elles se produisirent de manière presque continuelles, elles contribuèrent à faire de la période 1800-1814 une époque d'enlisement, au bord de la dépression démographique, comme nous aurons l'occasion de le vérifier. 


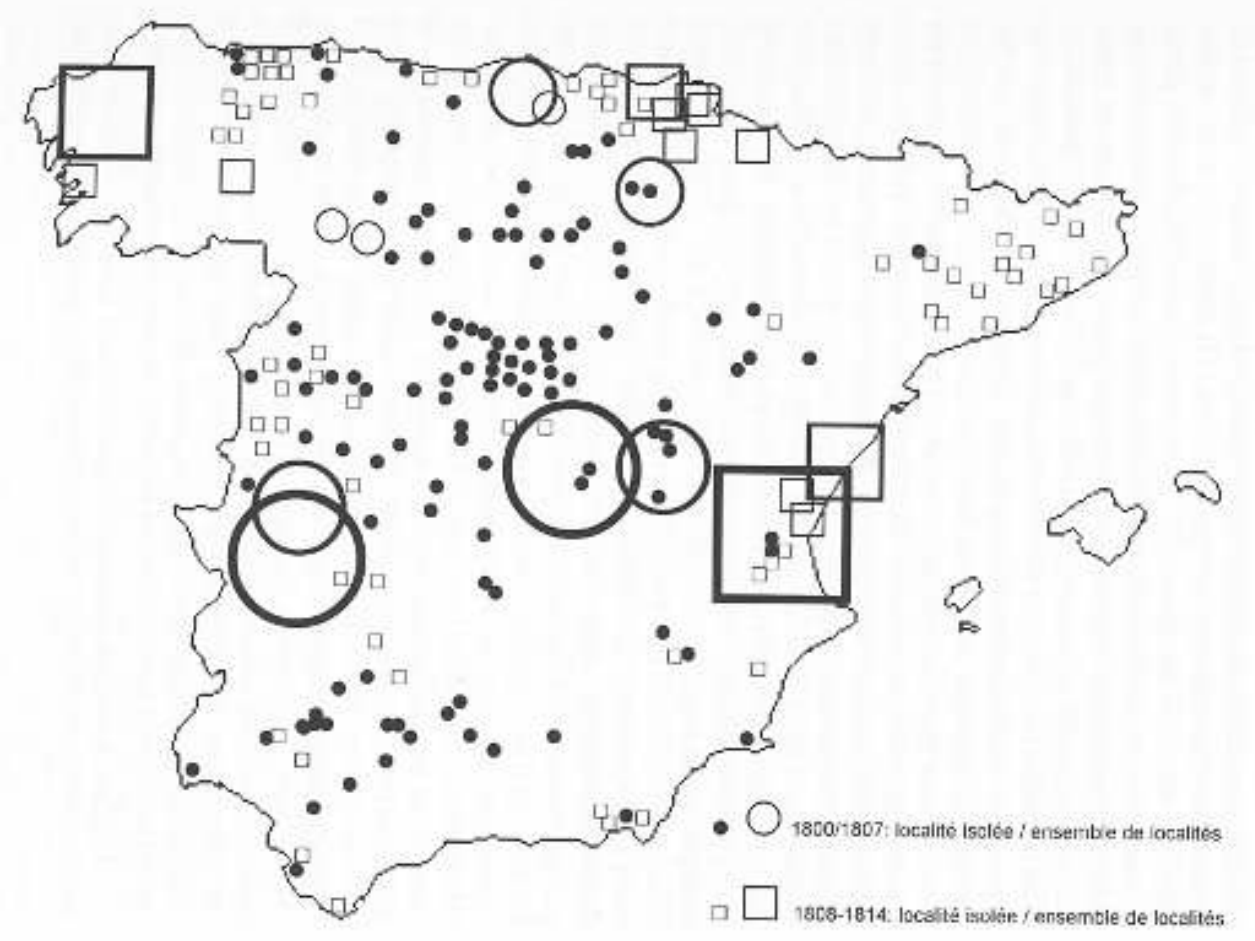

Jusqu'à présent, nous avons analysé la profondeur de la crise de la guerre en parallèle avec celle du début de siècle, et identifié durant celle-ci les années où la mortalité fut la plus élevée, en tentant d'expliquer le phénomène. Nous pouvons dire que la Guerre d'Indépendance ne fut pas en elle-même une crise démographique de dimension exceptionnelle, mais plutôt le deuxième épisode d'une crise plus étendue, débutant avec le changement de siècle, et dont la première partie se déroula quelques années auparavant ; nous pouvons également affirmer que les années où la mortalité fut la plus élevée -dans la mesure où celle-ci fut liée à la crise de subsistance et aux épidémies- ne doivent être attribuées au conflit armé que de manière indirecte et que les victimes se trouvèrent probablement en plus grand nombre parmi la population civile que parmi les combattants. Ces dernières affirmations méritent un éclaircissement supplémentaire. Nous savons que certains épisodes de la guerre qui, de par leurs répercussions, pourraient laisser à penser qu'ils furent l'occasion de véritables boucheries, n'occasionnèrent qu'une mortalité limitée : à Gérone en 1809, année du troisième et du plus sanglant siège de la ville, périrent un peu plus de 1500 personnes, chiffre qui n'atteint même pas le double si l'on ajoute les militaires et la population non résidente ${ }^{14}$; dans Tarragone, assiégée et prise par les troupes de Suchet en 1811, les analyses les plus fondées évoquent un peu plus de 350 victimes civiles, soit un chiffre nettement inférieur aux 2100 décès subis durant la terrible année $1809^{15}$; SaintSébastien qui, dans l'été 1813, subit le siège, l'assaut et l'incendie par des troupes angloportugaises, perdit tout au plus 1200 personnes $^{16}$. Et bien qu'il soit difficile de distinguer entre population civile et militaire dans une guerre où le combat irrégulier éveillait plus d'enthousiasme que la lutte dans les rangs de l'armée, ni les limites atteintes par ce combat, ni son endurance devant l'assaut de l'armée napoléonienne la plus expérimentée ne permettent de croire à un pourcentage de perte élevé au champ de bataille: dans la cas de la région de Valdeorras, dans la province galicienne 
d'Orense, où pendant le soulèvement populaire de 1809 contre l'occupation française très peu de soldats moururent et à peine un neuvième des enterrements fut causé par la mortalité de guerre ${ }^{17}$, les proportions peuvent sembler extrèmes, mais pas irréelles dans un conflit où les combattants irréguliers, usant de méthodes proches d'une guérilla, offraient peu de chances de servir de cibles à leurs adversaires.

11 Il existe aussi des signes clairs du caractère inégal de l'impact de la guerre dans les différentes régions. Nous allons préciser ce dernier trait en nous concentrant sur les quatre régions pour lesquelles existent des séries de haute qualité et représentatives : la région de Cantabria, pour laquelle nous disposons d'un échantillon de 40 paroisses rurales, ce qui représente $16,8 \%$ de la population de la région ${ }^{18}$; Castellion, avec 40 paroisses dont la population représente $14,45 \%$ de la province ${ }^{19}$; l'ancienne région de Nouvelle-Castille (Madrid, Tolède, Ciudad Real, Cuenca et Guadalajara), pour laquelle Reher a confectionné une série à partir de données concernant 26 localités représentant $4,5 \%$ des habitants ${ }^{20}$, coïncidant avec celle que ce même auteur avait établie pour une zo ne plus petite de cette région (19 villages de la province de Cuenca $^{21}$; et la Catalogne, avec 98 séries dispersées dans toute la Principauté, ce qui équivaut à $14,6 \%$ de la population catalane ${ }^{22}$. Il existe des différences notables dans la croissance naturelle de chacune de ces régions pendant la période centrale de la guerre (1809-1813) : la Catalogne figure à une extrémité, avec une perte de $5 \%$ de la population, et la province de Castellion à l'autre extrémité, avec une croissance naturelle, tandis que les pertes de Cantabria et de la Nouvelle-Castille sont modérées. La dimension démographique de la guerre fut, selon ces données, très inégale dans les cadres divers de l'Espagne péninsulaire ; la remarque rejoint les observations faites lors de certaines crises démographiques antérieures, mais n'est habituellement pas soulignée dans l'histoire générale de la Guerre d'Indépendance. Cette dernière occasionna des degrés très variés de pertes humaines, et probablement de souffrances et de destructions matérielles, que ce soit dans l'une ou l'autre de ces régions. Et la Catalogne, d'après ces données, a été particulièrement affectée par le conflit.

\begin{tabular}{|l||l|l|l|l|}
\hline Croissance naturelle (\%) & Catalogne & Nouvelle-Castille & Cantabria & Castellion \\
\hline \hline 1809 & $-51,3$ & $-23,1$ & $-16,5$ & $-4,9$ \\
\hline \hline 1810 & $-5,4$ & 4,1 & 2,1 & 12,4 \\
\hline \hline 1811 & 11,4 & 15,1 & $-4,2$ & 8,7 \\
\hline \hline 1812 & $-16,1$ & $-9,3$ & $-2,4$ & 10,3 \\
\hline \hline 1813 & 11,6 & $-4,1$ & $-8,7$ & $-7,3$ \\
\hline \hline Cumulée $1809-1813$ (\%o) & $-49,8$ & $-17,3$ & 28,7 & 19,2 \\
\hline \hline Cumulée $1809-1813$ (personnes) & -54126 & -19280 & -4515 & 3591 \\
\hline
\end{tabular}

Ces années là, la natalité et la mortalité ont tendance à coïncider dans une trajectoire identique, responsable du signe négatif de la croissance naturelle dans la plupart des 
régions étudiées. Ainsi, en plus des décès, la guerre s'est aussi caractérisée par l'abscence de naissances. Si l'on prend comme point de référence les années précédant immédiatement le début du siècle (1787-1799), période de prospérité dans ces quatre régions -malgré la crise qu'a connu le nord-est de la Catalogne pendant la guerre de la Convention (1793-1795)-, les années centrales de la guerre (1809-1813) présentent un bilan unanimement négatif, avec des reculs généralisés du taux de natalité (TN) et une augmentation tout aussi généralisée du taux de mortalité (TM) :

\begin{tabular}{|l||l|l|l|l|}
\hline & Catalogne $^{23}$ & Nouvelle-Castille & Cantabria & Castellion \\
\hline \hline TN 1787-1799 (\%) & 50,12 & 40,97 & 34,73 & 42,36 \\
\hline \hline TN 1809-1813 (\%) & 46,70 & 36,83 & 28,61 & 37,24 \\
\hline \hline TM 1787-1799 (\%) & 37,84 & 32,20 & 30,91 & 30,07 \\
\hline \hline TM 1809-1813 (\%) & 58,84 & 40,35 & 34,54 & 33,41 \\
\hline \hline $\begin{array}{l}\text { \% de recul attribuable à } \\
\text { l'augmentation du TM }\end{array}$ & 86 & 66 & 37 & 39 \\
\hline
\end{tabular}

13 Au-delà de l'incertitude de quelques chiffres, notamment dans le cas catalan où leur niveau improbable est presque certainement dû à une dissimulation à la source du recensement de 1787, il convient d'observer l'aggravation des chiffres de la mortalité et de la natalité dans des proportions similaires, sauf là où la mortalité s'emballe pendant les années de guerre (Catalogne)

Graphique 2. Évaluation de l'évolution de la population espagnole, 1787-1815²4

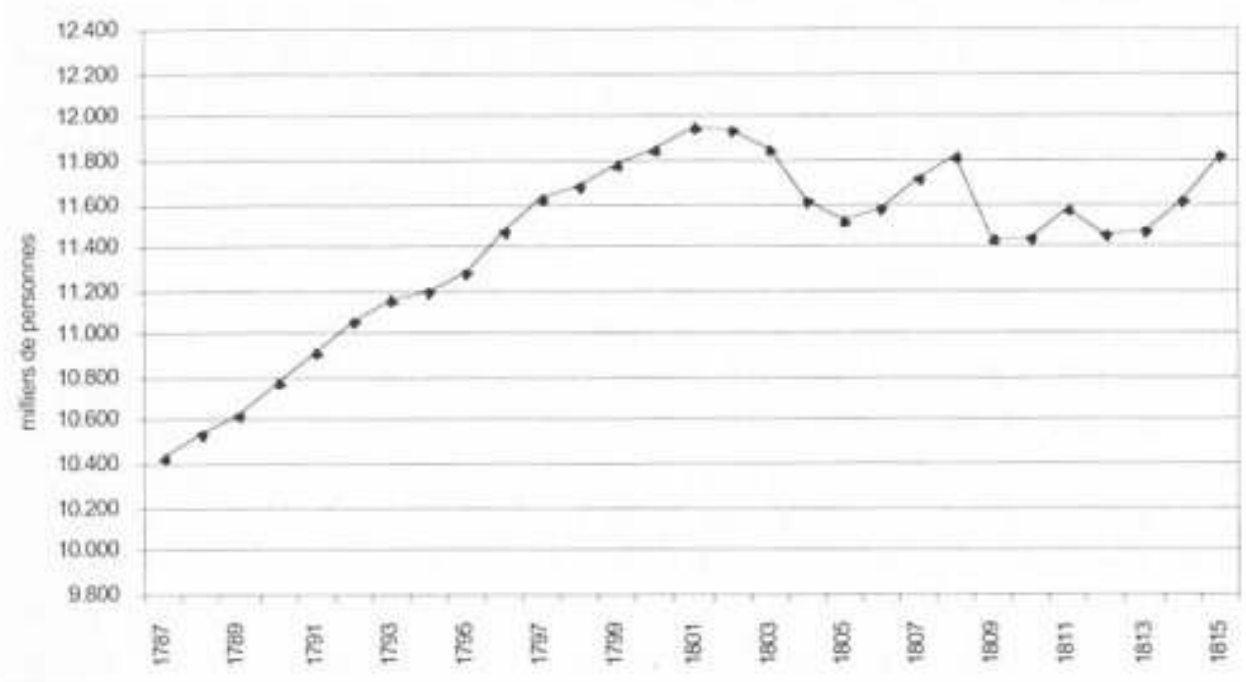

Mais apprécier l'ampleur de la baisse de la population pendant la guerre n'est pas toujours chose facile. Le graphique 2 représente une évaluation de l'évolution de la population entre 1787 et 1815, obtenue à partir de données pondérées des quatre séries 
régionales dont on a déjà parlée ${ }^{25}$. Le fait que la plus importante de ces séries - par son poids spécifique- (Nouvelle-Castille), se situe sur le plateau de Castille, semble bien contrebalancer le caractère périphérique et plutôt septentrional des trois restantes. En même temps, le caractère extrème de la crise des années de guerre en Catalogne n'est pas complétement exceptionnel dans l'histoire espagnole (voir graphique 1) et parait nuancé par l'impact plus léger qu'eut la crise dans les autres zones étudiées. C'est pourquoi nous pouvons, du moins provisoirement, accepter ces résultats comme représentatifs pour l'ensemble du territoire espagnol. En accord avec ceux-ci, la tendance à l'augmentation de la population du pays, qui gagne un million et demi d'hommes entre 1787 et 1801, s'inverse brusquement au cours des années suivantes, puisque s'observe une perte cumulée de presque un demi-million d'habitants; cette baisse se poursuivit et même s'aggrava légèrement pendant la plupart des années de guerre, avant un rapide recouvrement débutant en 1814. Dans son ensemble, quinze années furent gaspillées en ce début de siècle, dont la moitié coöncide avec la Guerre d'Indépendance, qui cumule encore un autre demi-million de morts en deux périodes successives (1809: presq ue 400 000personnes; 1812 : plus de 100 000), ce qui ralentit le recouvrement. Ces données servent à situer l'incidence de cette longue crise, qui commença en début de siècle et reprit pendant les années de guerre, dans la croissance de longue durée de la population espagnole. Mais elles ne permettent pas de mesurer, dans leur pleine dimension, les pertes causées par la guerre, ni leurs répercussions démographiques.

Il n'est pas facile de connaître la mortalité provoquée, directement ou non, par la guerre. Nous pourrions tenter de lui attribuer une proportion du demi-million d'habitants disparus après les deux crises de 1809 et 1812 ; deux tiers est un pourcentage plausible, ce qui nous donnerait un chiffre tournant autour de 350000 victimes. De façon un peu plus sophistiquée, mais pas pour autant plus précise, nous pourrions aussi comparer le taux de mortalité existant dans les quatre régions étudiées pendant les années 1809-1813, en pleine guerre, avec le taux de mortalité des années précédant la crise de début de siècle (1787-1799), puis mesurer - en nombre de personnes- la différence entre l'un et l'autre (165511) et appliquer le calcul à l'ensemble de la population espagnole (11 475 915, moyenne de 1809-1813). L'opération fait apparaître un excès de 735472 morts par rapport au nombre de décès qu'aurait provoqué le taux de mortalité de la période 1787-1799 ; cela dit, nous ne savons pas quelle aurait été la "mortalité normale » à prendre comme point de référence! $\mathrm{Ce}$ même calcul, prenant pour base de comparaison la période 1787-1807, qui inclue la crise du début de siècle dans le centre de l'Espagne, donne un chiffre de 586281 morts supplémentaires lors des années du milieu de la guerre (1809-1813). L'un et l'autre de ces chiffres sont très élevés, ce qui permet de supposer que même si l'apport de nouvelles séries nous permettait d'intégrer dans l'estimation des régions moins affectées par la guerre, les chiffres, toujours approximatifs, ne seraient pas très éloignés du demi-million de morts ${ }^{26}$. Ce qui est clair, en revanche, c'est que la Guerre d'Indépendance fut la plus coûteuse en vies humaines de toutes les guerres de l'histoire contemporaine espagnole, dépassant la Guerre Civile en mortalité relative : le demimillion de morts tombés durant cette dernière et son après-guerre immédiate représente moins de $2 \%$ des 26 millions d'Espagnols vivant alors ${ }^{27}$, tandis que les victimes du conflit de 1808-1814, en retenant l'hypothèse la plus conservatrice, dépasseraient largement les $2 \%$, pouvant même monter jusqu'à environ $5 \%$ des 11,5 millions d'habitants du pays. Et tout cela sans prendre en compte les pertes des armées 
napoléoniennes et alliées, qui auraient pu atteindre le quart de million, et dont la plupart appartiendraient aux forces françaises ${ }^{28}$. Il est très probable qu'aucun autre État européen de bonne dimension n'ait connu un tel bain de sang pendant l'époque napoléonienne. La France n'en connut pas de tel, bien sûr, et ce malgré l'importance des pertes militaires éprouvées pendant les guerres contre les coalitions successives ${ }^{29}$.

Une dernière question à prendre en considération est l'incidence qu'eut la Guerre d'Indépendance sur l'évolution postérieure de la population espagnole. La guerre n'a pas seulement donné lieu à une crise de mortalité, mais aussi à une baisse de naissances : dans presque toutes les régions où l'on connaît le taux de natalité, il connut alors une baisse notable, d'environ quatre à cinq points dans les séries correspondant à la période 1787-1799 étudiées ici, ce qui suggère une réduction voisine du tiers du surplus de mortalité, proportion qui semble beaucoup plus équilibrée et même inversée dans les régions où il n'y eut point de pointe de mortalité prononcée. Le maintien des taux moyens de natalité et de mortalité de la période mentionnée sur l'ensemble du pays aurait conduit la population espagnole en 1814 à un chiffre probablement supérieur d'un million de personnes, ou d'un peu plus de 700000 si l'on prend comme référence les taux de la période large de 1787-1807. Mais nous voici à nouveau face à des calculs théoriques, car dans des sociétés à l'économie pré-industrielle, les limites imposées à la croissance de la population par les ressources disponibles empêchent sa continuité. Quoi qu'il en soit, les crises successives du début du XIX ${ }^{\mathrm{e}}$ siècle produisirent un ralentissement de quinze ans dans la croissance que connut la population pendant le siècle précédent, même s'il est certain que la reprise, à partir de 1815, parvint à panser en très peu d'années les blessures subies. En revanche, l'effet combiné de la réduction de la natalité et de l'augmentation de la mortalité a bien créé l'apparition d'une génération vide, encore visible dans les brèches laissées sur les pyramides des âges un demi-siècle plus tard; on l'observe aussi (cf. graphique 3) dans le soudain abaissement des groupes d'âge supérieurs à 40 ans, dont le poids relatif a baissé de $20 \%$ par rapport au poids qu'ils avaient dans le recensement de 1797. Cette vague se propage tout au long de la deuxième moitié du XIX ${ }^{\mathrm{e}}$ siècle, chaque fois plus faiblement, sous la forme de générations successives diminuées ${ }^{30}$. 
Graphique 3. Distribution de la population par âges : comparaison entre 1857 et $1797^{31}$

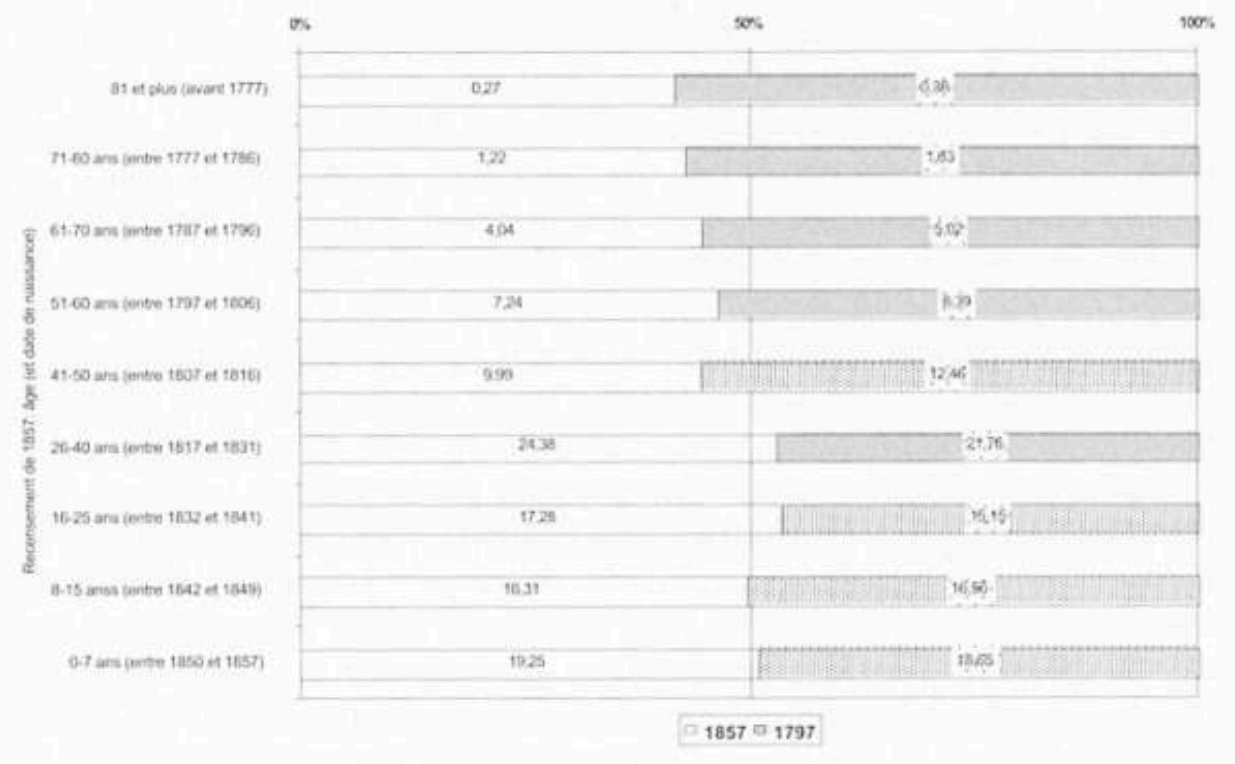

\section{NOTES}

1.Un premier état de cette recherche a été publié sous le titre «El impacto demográfico de la Guerra de la Independencia », dans Enfrontaments civils: postguerres i reconstruccions, Lleida, 2002, vol. 1, pp. 283-299.

2.Les sources de la carte 1 (voir page suivante), ainsi que celles indiquées dans l'appendice 2 du travail cité dans la note précédente, sont les suivantes : Francisco Ramírez, Comportamientos demográficos diferenciales en el pasado. Aplicación del método de reconstrucción de familias a la población de Iznájar, Grenade, 2001 (Iznájar se trouve en Andalousie); Manuel Jesús Fernández, « El final del Antiguo Régimen demográfico en Écija. El caso de Santa María (1800-1860) », dans Actas del V Congreso «Écija en la Edad Contemporánea " ,Écija, 2000, pp. 337-349 (Écija, en Andalousie); Jorge Sánchez, Valladolid durante la Guerra de la Independencia española, 1808-1814, Valladolid, 2002 ; Josep Ma Planes, Demografia i societat de Tàrrega durant l'Antic Règim, thèse doctorale 1987, Université de Barcelone, Microfitxa, Publicacions Universitat de Barcelona, 1988 (Tàrrega, en Catalogne); P. Erdozain - F. Mikelarena, « Evolución demográfica y crisis de mortalidad en las Cinco Villas de la montaña navarra entre 1700 y 1860 », Revista de Demografía Histórica, XX, 1, 2002, pp. 145-177 (région des Cinco Villas, en Navarre); données fournies par Antonio Prada pour un ensemble de 25 paroisses de Guipúzcoa (Pays Basque) : Arrasate-Mondragón, Azkoitia, Azpeitia, Beasain, Bergara (Sta. Maria), Bergara (S. Pedro), Bidania, Errentería, Hondarribia, Irún, Itsaso, Lazkao, Leaburu, Legazpi, Mutriku, Oñati, Ordizia, Orio, Pasajes de S. Juan, Segura (Sta Ma), Soraluze, Tolosa, Urretxu, Zarautz, Zumarraga; Joan Serafí Bernat (ed.), Les sèries vitals valencianes, vol. 1. Les comarques septentrionals, Valence, 1996 (20 localités de la province de 
Castellion, à Valence : L'Alcora, Atzeneta del Maestrat, Betxí, Castellfort, Cortes de Arenoso, Costur, Culla, Forcall, La Llosa de la Plana, Mascarell, Nules, Orpesa, Ortells, Palanques, Petrés, La Serratella, La Serra d'en Galceran, Vall de Almonacid, Vilafranca, Vinaròs).

3.Isidro Dubert, « La mortalité en Galice, 1600-1850 », Annales de Démographie Historique, 1, 1996, pp. 221-248.

4.Ramon Alberch, « La demografía gironina del 1808 al 1814 », Estudi General, 1, 1981, pp. 173-178.

5.Josep M. Benaul, Guerra i canvi econòmic. L'impacte de la Guerra del francès en la industria tèxtil llanera de Sabadell i Terrassa, 1808-1914, Sabadell, 1993.

6. Maties Ramisa, La Guerra del francès al corregiment de Vic: 1808-1814, Vic, 1993.

7.Ramón Lanza, La población y el crecimiento económico de Cantabria en el Antiguo Régimen, Madrid, 1991, p. 276.

8.Gonzalo Anes, Las crisis agrarias en la España moderna, Madrid, 1970, graphiques 42-47. 9.J. S. Bernat et M. A. Badenes, «Cronología, intensidad y extensión de las crisis demográficas en el País Valencià (siglos XVII-XIX) », dans Estudis sobre la població del País Valencià, Valence, 1988, vol. 1, pp. 537-557.

10.Manuel Espadas, «El hambre de 1812 en Madrid », Hispania, 1968, pp. 594-623; María F. Carbajo, La población de la villa de Madrid desde finales del siglo XVI hasta mediados del siglo XIX, Madrid, 1987.

11.Rafael Torres Sánchez, Aproximación a las crisis demográficas en la periferia peninsular. Las crisis en Cartagena durante la edad moderna, Carthagène, 1990; Diego Sánchez Jara, Intervención de Murcia en la Guerra por la Independencia, Murcie, 1960.

12.Sources: Cantabria: Lanza 1991, à partir de TM ; Castilla la Nueva: David S. Reher, « Dinámicas demográficas en Castilla la Nueva, 1550-1900: un ensayo de reconstrucción ", dans Actas del II Congreso de Demografía Histórica, vol. III, Alicante, 1991, pp. 17-75, à partir du taux de mortalité (TM) ; Catalogne: 67 localités (Jordi Nadal, « Las grandes crisis de mortalidad de los años 1793-1812: los efectos a largo plazo en la población catalana », Boletín de la Asociación de Demografía Histórica, VIII, 2, 1990, pp. 37-49, 13 paroisses; A. Moreno - L. Navarro, « La población de Reus [corregimiento de Tarragona] durante la Guerra de la Independencia ", dans La Guerra de la Independencia [1808-1814] y su momento histórico, Santander, 1982, I, pp. 161-163, Reus; 53 séries indiquées dans la note 23), à partir de chiffres absolus; Castille-Léon: Pablo García Colmenares, Estancamiento demográfico y estabilidad social en Castilla (1750-1930), Valladolid, 1998 (Ville de Palencia), Ángel García Sanz, Desarrollo y crisis del Antiguo Régimen en Castilla la Vieja, Madrid, 1986 (14 localités de Ségovie), Ricardo Robledo, Historia de Salamanca. Tomo IV. Siglo XIX, Salamanque, 2001 (Aldehuela de la Bóveda), Laureano M. Rubio, La Bañeza y su tierra, 1650-1850, un modelo de sociedad leonesa, Léon, 1987 (région de La Bañeza), José Luis Sáinz - Francisco J. Sanz, « Evolución demográfica del partido de Candemuño (1700-1850). Apuntes a la mortalidad catastrófica ", El pasado histórico de Castilla y León, vol. II: Edad Moderna, Burgos, 1983, pp. 355-379 (Cabia et Mahamud), à partir de chiffres absolus (pour La Bañeza, estimés à partir de graphiques); Cáceres: Miguel Ángel Melón, Extremadura en el Antiguo Régimen. Economía y sociedad en tierras de Cáceres, Mérida, 1989 (15 paroisses), à partir de graphiques de taux; Asturies: Carmen Ma Sanzo, « La población de Asturias en los siglos XVII a XIX: los registros parroquiales », La economía española al final de Antiguo Régimen. I. Agricultura, Madrid, Alianza, 1982, pp. 259-343 (20 localités), à partir de chiffres absolus; Madrid: María F. Carbajo, La población de la villa de Madrid desde finales del siglo XVI hasta mediados del siglo XIX, Madrid, 1987 (ensemble de 
paroisses de la ville), à partir de taux; Andalousie: 22 localités, dont 17 paroisses de la ville de Séville, à partir de chiffres absolus (références indiquées dans les cartes 1 et 2; Guipúzcoa: à partir de chiffres absolus des 25 paroisses comprises dans les cartes 1 et 2 (données fournies par Antonio Prada); Castellion: Bernat 1996, 20 localités décrites dans les cartes 1 et 2 , à partir de taux de mortalité.

13.Les sources utilisées dans cette carte, en plus de celles indiquées dans l'appendice 1 du travail cité dans la note 1, sont les suivantes: Ramírez 2001 (Iznájar, en Andalousie); Fernández 2000 (Écija, en Andalousie); Planes 1988 (Tàrrega, en Catalogne); Erdozain Mikelarena 2002 (région des Cinco Villas, en Navarre); données fournies par Antonio Prada pour un ensemble de 25 paroisses de Guipúzcoa (Pays Basque); Bernat 1996 (20 localités de la province de Castellion, à Valence).

14.Ramon Alberch, op. cit., p. 174.

15.José Ma Recasens, «La población de la ciudad de Tarragona durante la Guerra de la Independencia », dans Estudios de la Guerra de la Independencia, Zaragosse, 1964, vol. 1, pp. 485 et 479.

16.Juan Bautista Olaechea, ¿Quién destruyó San Sebastián?, Saint-Sébastien, 1973, p. 22. 17.Ramón López Caneda, Valdeorras en la Guerra de la Independencia, Barco de Valdeorras, 1989 , pp. 264 et 270.

18. Ramón Lanza, op. cit.

19.Joan Serafí Bernat (éd.), Les sèries vitals valencianes, vol. 1. Les comarques septentrionals, Valence, 1996.

20.David S. Reher, « Dinámicas demográficas ».

21.David S. Reher, « La crisis de 1804 y sus repercusiones demográficas: Cuenca (1775-1825) », Moneda y Crédito, n. 154, 1980, pp. 33-72.

22.Cette série conjointe a été établie en unissant la série de 45 paroisses catalanes (équivalentes à 9,3\% de la population) élaborée par Jordi Nadal (La población española, Barcelone, 1986, p. 129), les données des 53 localités suivantes: S. Quintí de Mediona, Sta Ma de Mediona, Gelida, Pacs, St. Llorenç d'Hortons, Vilanova (St. Antoni), La Geltrú (Santa Ma), Monistrol d'Anoia, Vilobí, Vilafranca del Penedès, Santa Margarida i els Monjos, fournies par Francesc Munõz, Creixement demogràfic, mortalitat i nupcialitat al Penedès (segles XVII-XIX), Thèse Doctorale, UAB, 1990; Aguiló, Barberà de la Conca, Belltall, Conesa, Espluga de Francolí, Lilla, Passanant, Rocafort de Queralt, Rojals, Santa Coloma de Queralt, Savellà del Comtat, Segura, Solivella, Vallclara, Vilanova de Prades, Vilaverd-La Riba, Vimbodí, empruntées à Valentí Gual, Balanç natural i reconstrucció de families a travès dels sacramentaris: la Conca de Barberà a l'Època Moderna, Thèse Doctorale, Université de Barcelone, 1991 (Microfitxa, Publicacions Universitat de Barcelona, 1992); Agullana, Borrassà, Cabanes, Castelló d'Empúries, L'Escala-St. Martí d'Empúries, La Junquera, Navata, Ordis, Roses, Ventalló i Valveralla, recueillies de Miquel Planas, $L a$ població a l'Alt Empordà al règim demogràfic antic, Thèse de licence, UAB, 1985; Botarell, Bràfim, El Catllar, Cornudella, Els Garidells, Perafort, La Pobla de Montornès, Poboleda, Pratdip, Riudecanyes, Riudecols, La Secuita, Torroja, Ulldemolins, dans Jordi Andreu, El Camp de Tarragona i el Priorat durant els segles XVIII $i$ XIX, Thèse Doctorale, UAB, 1994; Sant Salvador de Vendrell y Sant Vicenç de Calders, en Salvador Caralt, Evolució demogràfica del Vendrell. Segles XVI-XIX, Thèse de licence, UAB, 1986. Ces 53 séries rassemblaient 46977 habitants en 1787, soit 5,3\% de la totalité de la population catalane figurant dans le recensement de 1787. 
23.Pour cette comparaison, nous n'avons pu utiliser que les chiffres des 53 séries paroissiales énumérées dans la note précédente (équivalents à 5,3\% de la totalité de la population catalane).

24.Les valeurs du graphique sont les suivantes: 1787, $10427828 ; 1788,10543$ 715; 1789, 10631 446; 1790, 10 771 511; 1791, 10913 750; 1792, 11058 276; 1793, 11158 117; 1794, 11201 756; 1795, 11286 102; 1796, 11473 982; 1797, 11622 029; 1798, 11679 988; 1799, 11776 183; 1800, 11848 867; 1801, 11955 107; 1802, 11944 542; 1803, 11851 359; 1804, 11617 925; 1805, 11518 441; 1806, 11581 313; 1807, 11 719 680; 1808, 11808 693; 1809, 11428 880; 1810, 11439 201; 1811, 11575 399; 1812, 11459 895; 1813, 11476 200; 1814, 11611 326; 1815, 11824 370. Ces données étant recueillies à partir du recensement de 1787 dans les premiers mois de l'année, les 10409879 habitants recensés furent comptabilisés pour l'année 1786.

25.Suivant les chiffres du recensement de 1787, la somme des habitants des quatre régions atteignait 2298 360, parmi lesquels 1085251 appartenaient à la NouvelleCastille (47,22 \%), 886624 à la Catalogne (38,58\%), 170988 à Castellion (7,44\%) et 155497 au Cantabria (6,76 \%).

26.Une de ces régions devait être la région valencienne, pour laquelle J. S. Bernat et $\mathrm{M}$. A. Badenes ont évalué que la mortalité entre 1809 et 1813 effectua une contribution négative à la croissance de la population de l'ordre de 2,7 \%, un peu plus de 25000 personnes (« Muerte y comportamiento demográfico de los valencianos [siglos XVIIXIX] », Dans Josep Bernabeu [dir.], El papel de la mortalidad en la evolución de la población valenciana. Actas del II Congreso de la Asociación de Demografía Histórica, Alicante, 1991, vol. 5, pp. 27-46).

27.Juan Díez Nicolás, « La mortalidad en la Guerra Civil española », Boletín de la Asociación de Demografía Histórica, n. 1, 1985, pp. 41-50.

28. Esteban Canales, op. cit.

29.Pour les douze années comprises entre 1803 et 1814, période plus étendue que celle de la guerre en Espagne, Jacques Houdaille a calculé un chiffre de 440000 pour les pertes militaires françaises certaines, auxquelles il faudrait ajouter une quantité similaire de pertes incertaines ("Le problème des pertes de guerre », Revue d'Histoire Moderne et Contemporaine, tome XVII, 1970, pp. 411-423). La France comptait alors quelque 30 millions d'habitants, soit plus de deux fois et demie l'effectif de l'Espagne. La mortalité ne s'est pas non plus acharnée sur la population civile : les courbes de natalité et de mortalité recueillies dans le vol. 3 de l'Histoire de la population française dirigée par Jacques Dupâquier (Paris, 1998) présentent un bilan annuel presque unanimement positif durant cette période.

30.Jordi Nadal, La población española, op. cit., pp. 136-137; David Reher, « La crisis de 1804 », art. cit., p. 64.

31.La distribution par âges des recensements de 1797 et de 1857 figure dans: Roser Nicolau, « Población », et dans Albert Carreras (dir.), Estadísticas históricas de España. Siglos XIX-XX, Madrid, 1989, p. 68. 


\section{RÉSUMÉS}

L'article a pour objet l'étude des caractères et de l'importance de la crise provoquée par la guerre qui ravagea le territoire espagnol entre 1808 et 1814 ; il s'agit à la fois d'estimer les pertes humaines subies à l'époque du conflit, d'examiner leur distribution dans le temps et dans l'espace, et d'évaluer ainsi l'impact de ces années sur l'évolution de la population espagnole.

\section{8-1814 : Demography and War in Spain}

This article surveys the characteristics and importance of the crisis triggered by the war which ravaged the Spanish homeland between 1808 and 1814; the aim being to estimate attrition suffered during the conflict, examine the distribution of losses in time and space, and thus assess the impact of these years on the evolution of the Spanish population.

INDEX

Mots-clés : Espagne, Guerre de l'Indépendance, démographie, population, Guerre d'Espagne

\section{AUTEUR}

\section{ESTEBAN CANALES}

Universitat Autònoma de Barcelona - Departament d'Història Moderna i

Contemporània 